
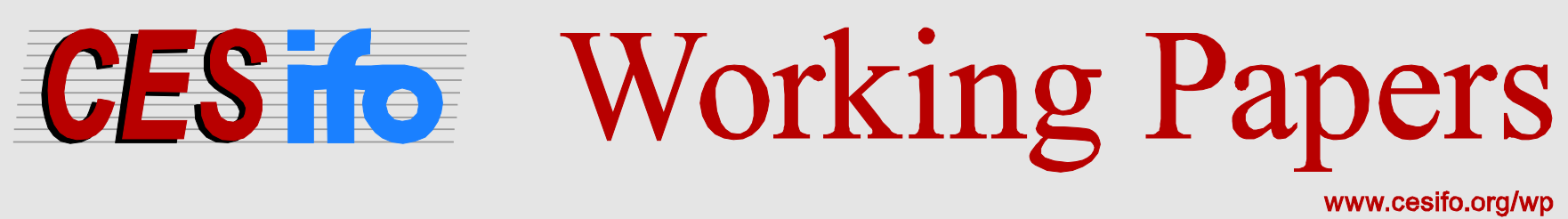

\title{
Measuring Health Inequality with Categorical Data: Some Regional Patterns
}

\author{
Joan Costa Font \\ Frank Cowell
}

\section{CESIFO WORKING PAPER NO. 4427}

CATEGORY 1: PUBLIC FinANCE

OCTOBER 2013

An electronic version of the paper may be downloaded

- from the SSRN website:

- from the RePEc website:

- from the CESifo website:

WWW.SSRN.com

Www.RePEc.org

www.CESifo-group.org/wp

\section{CESifo}




\title{
Measuring Health Inequality with Categorical Data: Some Regional Patterns
}

\begin{abstract}
Much of the theoretical literature on inequality assumes that the equalisand is a cardinal variable like income or wealth. However, health status is generally measured as a categorical variable expressing a qualitative order. Traditional solutions involve reclassifying the variable by means of qualitative models and relying on inequality measures that are mean independent. We argue that the way status is conceptualized has important theoretical implications for measurement as well as for policy analysis. We also bring to the data a recently proposed approach to measuring self-reported health inequality that meets both rigorous and practical considerations. We draw upon the World Health Survey data to examine alternative pragmatic methods for making health inequality comparisons. Findings suggest significant differences in health inequality measurement and that regional and country patterns of inequality orderings do not coincide with any reasonable categorization of countries by health system organization.
\end{abstract}

JEL-Code: D630, H230, I180.

Keywords: health inequality, categorical data, health surveys, upward status, downward status.

Joan Costa Font

Department of Social Policy

London School of Economics \& Political

Science ( LSE)

Houghton Street

UK - London WC2A 2AE

j.costa-font@lse.ac.uk
Frank Cowell

Department of Economics

London School of Economics \& Political

Science (LSE)

Houghton Street

UK - London WC2A 2AE

f.cowell@lse.ac.uk

July 2013 


\section{Introduction}

The measurement of inequality typically has three components: an equalisand (the thing that may be unequally distributed), the unit of analysis (the social or economic entities among whom the distribution takes place) and an aggregation method (the mathematical formula that assembles the information in the distribution). The first and third of these components can be tricky. If the equalisand is something that has an unambiguous cardinal representation and is intrinsically non-negative (consumption expenditure? hectares of land?) then the rest of the measurement problem is comparatively straightforward: you specify carefully whether the unit of analysis is the individual person, the household or something else, you choose the particular aggregation method that appropriately represents the inequality principles that you consider reasonable (an Atkinson index? the Gini coefficient?) and the problem is solved. If the equalisand is cardinally measurable but can take negative values (personal net worth?) then matters become trickier not all inequality measures are well defined in such cases and so you will be restricted in your choice of aggregation method. However, there may be a deeper problem: the equalisand itself may be a concept that is not naturally susceptible to representation on a cardinal scale. This is often the case when considering inequality of health status (Van Doorslaer and Jones 2003) or satisfaction (Oswald and Wu 2011, Stevenson and Wolfers 2008, Yang 2008). This measurability problem raises serious theoretical and practical difficulties when one wants to discuss the inequality in health status, which we will address in this paper, namely cardinalisation strategies and dealing with self-reported health correlation with other relevant covariates.

"Health inequality" suggests an approach to inequality that incorporates some concept of the hierarchy or status that covaries with health measures. Most of the world in epidemiology and health economics take a "materialistic approach" to inequality measurement whereby individual status refers to the individual position in the hierarchy of consumption or income (Marmot 2005, Wagstaff and van Doorslaer 2000). Therefore, policies that improve the distribution of material conditions are assumed to translate into fairer distribution of health status. The latter comes with some important caveats. First, health inequalities might not be the result of the status variable, and income as a status variable is itself a matter of choice and its use is problematic. Second, we ignore the proportion of social inequalities in health that are essentially "avoidable" or perhaps "ethically legitimate" (Fleurbaey and 
Schokkaert 2011, LeGrand 1987). For instance, inequalities resulting from the pure depreciation of health capital over time are arguably not avoidable; the same could apply to biologically or generically driven gender differences in health (Wagstaff et al. 1991).

The response to these difficulties has mainly been pragmatic. For example, the current mainstream literature on health inequality relies heavily on concentration indices (Costa-Font and Hernández-Quevedo 2012) and the use of categorical variables, but there is really very little in the literature that examines the assumptions that underpin the techniques that are commonly used. There are various types of cardinalisation methods that have been proposed in the literature - such as imputation, interval regression and so on but there is insufficient discussion of the economic rationale for these methods or the practical implications of using one method rather than another. Furthermore, such cardinalisation has been found to be the main source of bias: in a paper running a meta-regression of health inequality studies in the economics literature Costa-Font and Hernández-Quevedo (2013) find that the main reason for estimate heterogeneity is the way studies cardinalise the health status. As a consequence, one should treat the existing literature with some caution.

There is also a theoretical literature on the problem of making inequality comparisons when the underlying equalisand is ordinal, but it has mainly resulted in a number of rather limited propositions that are difficult to interpret or apply. However, recent work on the analysis of distributions of categorical variables has shown how natural interpretations of individual status can be used to provide a robust approach to the inequality-measurement problem in this context without resort to arbitrary cardinalisation of ordinal concepts (Cowell and Flachaire 2012). The status concept is similar to concepts used in poverty and relative deprivation and in recent approaches to the inequality of opportunity (de Barros et al. 2008). This gives rise to a new set of inequality indices that incorporate conventional distributional views such as degrees of inequality aversion and that can be applied to commonly used measures of well-being.

The results from this paper are the first outcomes of an attempt to identify a more precise definition of measures of health status. We expect our findings to provide researchers with a means of testing alternative ways of measuring inequalities of non-cardinal outcomes that may have significant policy implications. This is particularly important taking into account that measures of health inequality are used to rank health systems, and increas- 
ingly measures of well-being are used by the United Nations to evaluate institutions and public policies. Here we undertake the following:

1. We examine the extent to which health-inequality rankings are affected by alternative analytical approaches to the categorical-data problem, including the conventional cardinalisation methods and the CowellFlachaire status-inequality approach.

2. We provide a first estimate of well-being inequality rankings of world countries using the status-inequality approach.

3. We then examine whether patterns of inequalities in categorical measures health vary across different world regions. Do poor countries look different from medium / rich in terms of health-inequality rankings? Is there a regional consistency in inequality patterns?

The rest of the paper is organised as follows. Section 2 contains some necessary theoretical background, section 3 introduces the data set and explains our empirical strategy, section 4 contains our results and section 5 concludes.

\section{Approaches to inequality measurement with ordinal data}

There are two main approaches to the measurement of inequality using ordinal data. One could impute some artificial index of individual health status as a function of the categories. In some cases the imputation is achieved through subjective evaluation by individuals (for example on a Likert scale) and in some cases by making use of quality of life indices (for example the Quality-Adjusted Life Year). The same procedure can be applied to entities that do not have a natural ordering, such as vectors of attributes or endowments; one uses a utility function to force an ordering of the data. This is similar to one of the standard theoretical approaches to the measurement of multi-dimensional inequality - one computes the "utility" of factors and then computes inequality of utility, where the utility function is an appropriate aggregator (Maasoumi 1986, Tsui 1995). However the approach faces serious objections such as the arbitrariness of the cardinalisation, the arbitrariness of aggregating apples and oranges, and the arbitrariness of attempting to 
include measures of dispersion into the index as well. Even if the resulting well-being index appears reasonable over a wide subset of categories one might still be concerned about the way extreme values are represented in the index and their consequences for inequality comparisons.

A second approach to inequality developed within the health literature involves a reworking of traditional inequality-ranking approaches focusing on first-order dominance criteria - see Abul Naga and Yalcin (2008), Allison and Foster (2004), Zheng (2011). ${ }^{1}$ It is commonly suggested that the median could be used as an equality concept corresponding to the use of the mean in conventional inequality analysis, although it has been noted that comparing distributions with different medians raises special issues (Abul Naga and Yalcin 2010). But the approach runs into difficulty if quantiles are not welldefined, as may happen in the case of categorical variables - see Cowell and Flachaire (2012).

An alternative way forward introduced by Cowell and Flachaire (2012) tackles the problem by separating out carefully the two tricky components of inequality measurement mentioned in the introduction, the equalisand and the aggregation method. each of these is underpinned by an axiomatic argument that goes back to first principles. In short the resulting Cowell and Flachaire method amounts to an aggregation of the discrepancies between each person's actual status and some status reference point. In such an approach clearly a lot rests on the precise definition of status. In the case of applications where the equalisandum has a natural cardinalisation (income or wealth for example) then it makes sense to define status as income or wealth. However, where only ordinal information is available - as with categorical data on health status - then we have to do more. Suppose that information is purely categorical, in that we only know how many people are in each category $k=1,2, \ldots K$, but that the categories can be arranged in increasing order of their desirability. Then a simple argument shows that, if there are $n_{k}$ persons in category $k=1,2,3, \ldots K$, then the status of person $i$ who is currently in category $k(i)$ must be a function of either $\sum_{\ell=1}^{k(i)} n_{\ell}$, or $\sum_{\ell=k(i)}^{K} n_{\ell}{ }^{2}$ The first of these is a "downward looking" concept and the the second is its "upward looking" counterpart. It may be appropriate to normalise by the

\footnotetext{
${ }^{1}$ See also the similar polarisation approach developed by Apouey (2007).

${ }^{2}$ This is in line with recent proposal for a cardinalisation of Self-reported Health Status (SRHS) by applying an imputation of the values of Health-Related Quality of Life (HRQoL) values employed by Van Doorslaer and Jones (2003), Fonseca and Jones (2003). See section 3.3 below.
} 
size of the total population $n:=\sum_{1}^{K} n_{k}$ so that person $i$ 's status is given by either the downward-looking version

$$
s_{i}=\frac{1}{n} \sum_{\ell=1}^{k(i)} n_{\ell}
$$

or by the "upward-looking" counterpart of (1):

$$
s_{i}^{\prime}=\frac{1}{n} \sum_{\ell=k(i)}^{K} n_{\ell} ;
$$

On either definition status must lie between zero and one. If there were perfect equality (everyone in the same category) then it is clear that both (1) and (2) take the value 1 . It turns out that this, the maximum-status value, is the only thing that makes sense as the reference point. ${ }^{3}$

So the inequality-measurement problem then amounts to aggregating the information in the vector $\mathbf{s}:=\left(s_{1}, s_{2}, \ldots, s_{n}\right)$ in relation to the equality vector $(1,1, \ldots, 1)$. On the basis of some elementary axioms (Cowell and Flachaire) show that inequality must take the form of an index in the following family, indexed by $\alpha$ :

$$
I_{\alpha}(\mathbf{s})=\left\{\begin{array}{cl}
\frac{1}{\alpha(\alpha-1)}\left[\frac{1}{n} \sum_{i=1}^{n} s_{i}^{\alpha}-1\right], & \text { if } \alpha \neq 0 \\
-\frac{1}{n} \sum_{i=1}^{n} \log s_{i} . & \text { if } \alpha=0 .
\end{array}\right.
$$

where $\alpha$ is a parameter less than 1 . The family (3) is very similar to the well-known Generalised entropy class of inequality indices. The parameter $\alpha$ specifies the desired sensitivity of the index to a particular part of the income distribution: for low values of $\alpha$ the index $I_{\alpha}(\mathbf{s})$ is particularly sensitive to values of $s_{i}$ close to zero.

So we have a family of indices that is suitable for making comparisons of inequality in terms of health status. In what follows we shall suggest a way of using this to make health-inequality comparisons internationally.

\footnotetext{
${ }^{3}$ If you try to use the mean (of status) this does not remain constant under changes in the distribution and, as a consequence, produces counterintuitive behaviour of he index. The median is ambiguous in the case of categorical data. But the zero-inequality distribution just described is well-defined and always produces a well-behaved index.
} 


\section{Data and Methods}

The core of the analysis involves three main components: (1) extraction of suitable categorical variables on which to construct well-being indices; (2) computation of cardinal imputations, status measures and associated inequality indices and rankings; (3) an analysis of cross-country inequality comparisons.

\subsection{Data}

Our approach requires quantitative analysis of internationally comparable data that contain measures of health status. Accordingly the main data source to be used is the World Health Survey which contains data from seventy countries; it collects comparable multidimensional micro-data on income, employment education and health. There are two reasons for the choice of this data base: first, its great advantage for comparative work; second, its standardised world wide stricture can assist in examining cross country patterns across heterogeneous world regions that exhibits different levels of economic and social development.

The World Health Survey (WHS) is a general population survey, developed by WHO to address the need for reliable information and to cater to the increased attention to the role of health in economic and human development. The survey contains data from randomly selected adults (i.e. older than 18 years of age) who reside in seventy one countries who implemented household face-to-face surveys, computer assisted telephone interview, or computer-assisted personal interview in 2002. Sample sizes vary from 1,000 to 10,000 .

Our measure of health status is the standard measure of self-reported health widely used in the literature as described in Table 4 in the Appendix. As a measure, it suffers from cultural adaptation problems that make cross country comparison challenging, but it appears to be an adequate measure for computing within-country inequalities.

\subsection{Background}

It is possible to estimate a health production $h_{i}$ function for individual $i$ in the following kind of specification:

$$
h_{i}=\Phi\left(\mathbf{x}_{i}\right)+\varepsilon_{i}
$$


where $\mathbf{x}_{i}$ represents known determinants of health and $\varepsilon_{i}$ a random component. Estimating such a function is more challenging than one might imagine because health status is a latent variable that cannot be observed in full. Instead, we can only rely on proxy estimates. A growing literature that that takes as it point of departure Idler and Benyamini (1997) typically employs self reported measures of health as proxies for individuals health status. However, more recently a literature has developed to show that perception and observation might not necessarily match Sen (2002). The relevant variable, namely health status, can take the form of a censored (when quality of life measures are used), binary, ordered scale and interval variable depending on the underlying assumptions maintained by researchers.

In some exceptional circumstances, health status is measured using a censored continuous variable approach (for example when visual analogue scales are employed). If, instead of this, a binary approach is followed (for example, measuring morbidity of a certain condition), inequalities can be measured using a standard limited-dependent model such as a logistic regression techniques (Kunst and Mackenbach 1994). As a result, it might be argued, the odds ratio of the underlying social position variable could say something about the extent to which social position influences health status. In the first place, if the health variable allows an unambiguous ordering, then ordered probit models will take into account the structure of the data. So, by assuming an order and that it is possible to observe the variable health (a latent health variable) and the cut-off points, the probability of respondents' classifying themselves on a specific scale can modelled in the standard fashion. However, even where this is an improvement with respect to binary measures of health for the purposes of measuring health inequality, it is still difficult to interpret the meaning of a change in the order between scales of self-reported health status. An alternative way has been to obtain a linear index based on rescaling the ordered variable to obtain a normalised health index, such as in (Cutler and Richardson 1997). However, this still implies accepting some arbitrary assumptions on the value and distribution of a person's health status. Furthermore, the underlying reasons for an individual's categorisation into a specific health scale are still not accounted for. Therefore, some research claims that self reported health status can be interpreted instead as individual's categorisation into an interval, which can be ascertained by finding a link between self-reported measures of health and some health utility indices (Van Doorslaer and Jones 2003). This allows the use of interval regression to convert categories into continuous indexes (by 
running the predictions of a model with an acceptable goodness of the fit) which can then be used to compute measures of inequality. However, all the strategies followed in the literature are liable to be problematic.

\subsection{Empirical Strategies}

The first step is to select categorical variables for measuring health. It is usual for both national and international surveys to contain information on measures of self-reported health in categorical form. The literature adopts different strategies to measure such a latent variable from categorical responses, but there is no consensus nor theoretically sound strategy to deal with that specific feature. For example, in the in the data set used here, the World Health Survey, the categorical measure of health is based on the responses to the question "how would you rate your health today?". This yields a personal evaluation of overall health with potential responses in five categories ranging from "very good" to "very bad" - see the Appendix for more detail.

In this paper we draw upon the Cowell and Flachaire (2012) methodology to undertake international comparisons of inequality of self reported health status. This is an alternative to cardinalisation proposals include the imputation of values from the Health-Related Quality of Life scales as in Van Doorslaer and Jones (2003), Fonseca and Jones (2003). Following Van Doorslaer and Jones (2003), the equivalent cardinal value of the cut-off point of each response to the ordinal question was obtained so as to estimate the cardinal value of self-reported health using an interval-regression approach. Alternatively, the common cardinalisation strategy includes using either ordinal or interval regression (Wagstaff and Van Doorslaer 1994). However, even when then methods are statistically valid, they impose non-neutral assumptions that are not theoretically grounded. Indeed, some studies estimate cardinal health status measures using a linear index based on rescaling the ordered variable to obtain a normalised health index, as in Cutler and Richardson (1997). This still implies accepting some arbitrary assumptions on the value and distribution of individual health status.In order to carry out our methodological comparison we will also employ those techniques.

In the case of categorical data a simple way to process the data is to rank the values underlying the latent variable health. But in doing so the real distance between categories is unknown. Furthermore, given the multiplicity of survey data, scales tend to be arbitrary. Ordinal regression is used with or- 
dinal dependent variables, and uses the logit/probit link function. However, ordinal regression requires the assumption that the effect of the independent variables is the same for each level of the dependent variable. Then model outcomes can be interpreted to exhibit an interval censoring. In other words, we observe the ordered category into which each observation falls, but not the exact value of the observation. Interval regression is a generalization of censored regression. Both ordered and interval regressions models can be used to then transform a categorical outcome into a continuous variable based on the parameters of the regression. However, the transformation is highly dependent in the covariates of the regression and on the arbitrary nature of different variable categories. The strategy we pursue here addresses this latter point and provides an alternative cardinalisation method, that we argue is more suitable to measure inequalities in health. The Cowell and Flachaire (2012) paper includes the derivation of the statistical properties of the class of inequality indices that emerge from the status-inequality approach.

\subsection{Our Approach}

Our approach in this paper is to identify patterns arising from the WHS international data set using a robust methodology that takes account of the categorical nature of the data and the problems of making comparisons between countries. This involves two steps:

1. We use the Cowell and Flachaire (2012) class of measures for a variety of values of the sensitivity parameter $\alpha$. In this way we avoid problems associated with arbitrary cardinalisation of the underlying categorical variables and allow some flexibility in the choice of inequality measure.

2. Bearing in mind that the data are based on subjective evaluation it is important to avoid problems that may arise from systematic response bias between countries (some progress has been made in using anchoring vignettes that is increasingly used to correct for this type of bias see Kapteyn et al. (2007) and Rice et al. (2012)). Specifically, median categories are regarded as not informative given that some countries habitually over-report. The term "moderate health" means different things across countries because people's expectations are different. For this reason we focus on inequality orderings within the WHS sample as well as inequality levels. 
3. Furthermore we examine possible patterns of health inequality by looking at the way in which (i) $I_{\alpha}(\mathbf{s})$ for each country varies and (ii) the way country orderings change as the parameter $\alpha$ varies.

4. Finally, we use rank correlation analysis to examine the association of country orderings when we vary the way status is computed, alongside changes in the values of the sensitivity parameter $\alpha$. A high association between different ordering and measures of status would be indicative of a limited effect of those two variables. In addition, identifying the values of $\alpha$ and status that yield higher correlation coefficients is of some interest as a sensitivity analysis.

\section{Results}

So, let us apply the status-based inequality measure (3) to the WHS data using the two definitions of status given in (1) and (2). Figures 1 and 2 show the relationship between $I_{\alpha}(\mathbf{s})$ and $\alpha$ for downward-looking and upwardlooking status respectively for eight OECD countries; a close-up version of the these figures (for $-1 / 2 \leq \alpha \leq+1 / 2$ ) is shown in Figures 3 and 4 .

The patterns illustrated in Figures 1 to 4 are typical. At a first pass it is clear that if we restrict attention to moderate values of the sensitivity parameter $(-1 / 2 \leq \alpha \leq+1 / 2)$ some of the conventional wisdom about OECD countries is confirmed: in the downward-looking case we can see that both Finland and France enjoy low health-status inequality. It is also clear that there is a difference between downward and upward-looking measures of status: compare the charts for neighbours Austria and Germany. However, if we allow versions of the inequality measure that are very sensitive to low status $(\alpha<-1 / 2)$ it is clear that the inequality profiles fan out dramatically and the conventional wisdom breaks down: within the OECD countries of WHS Finland and France are no longer low-inequality countries for this range of $\alpha$.

\subsection{Differences in upward and downward looking status}

As explained in section 3.4, we are interested in examining how rank order correlation vary when upward and downward status measures are used. To do so, we make use of graphical methods and correlation analysis. 
Figure 1: OECD inequality and $\alpha$. Downward-looking status

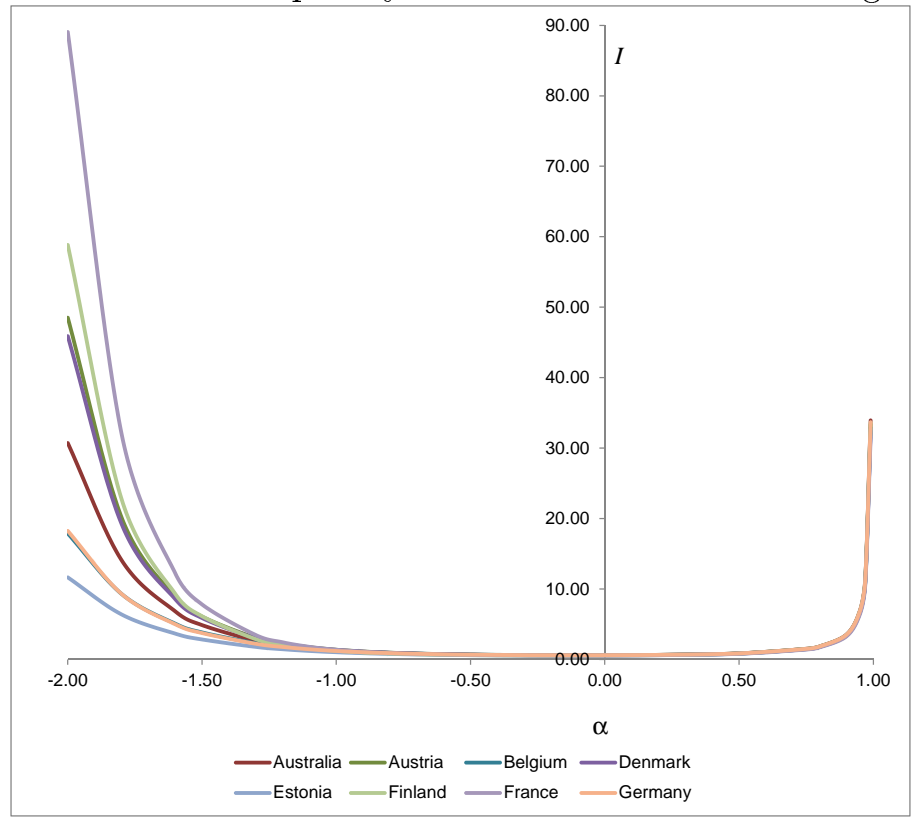

Figure 2: OECD inequality and $\alpha$. Upward-looking status




Figure 3: OECD inequality and $\alpha$. Downward-looking status (Close-up)

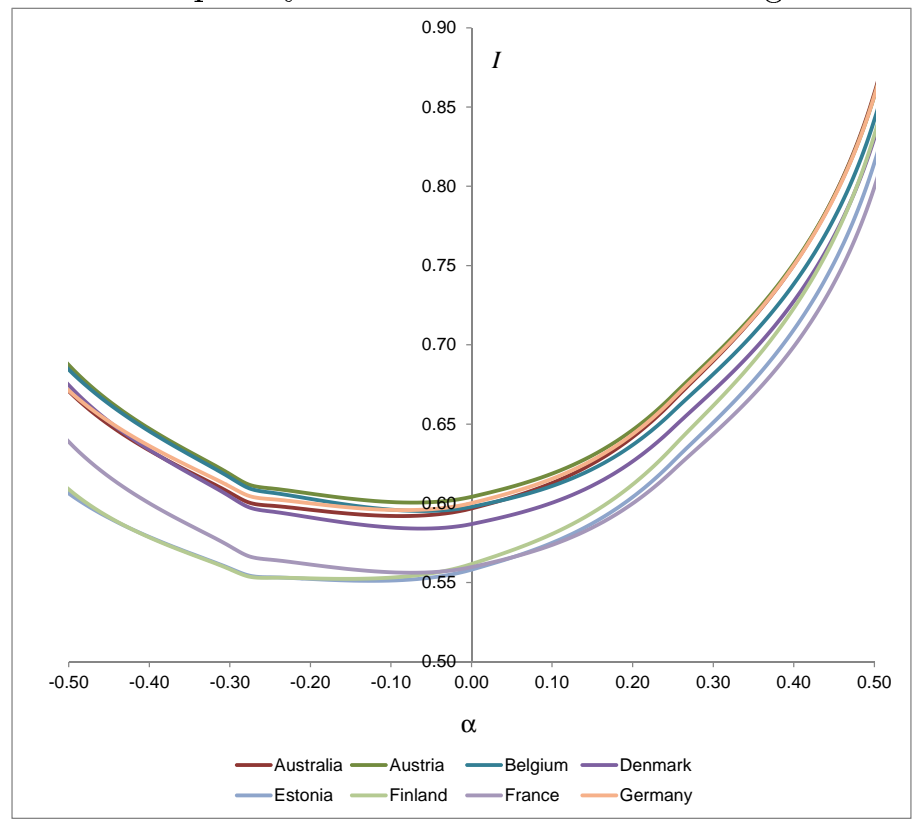

Figure 4: OECD inequality and $\alpha$. Upward-looking status (Close-up)

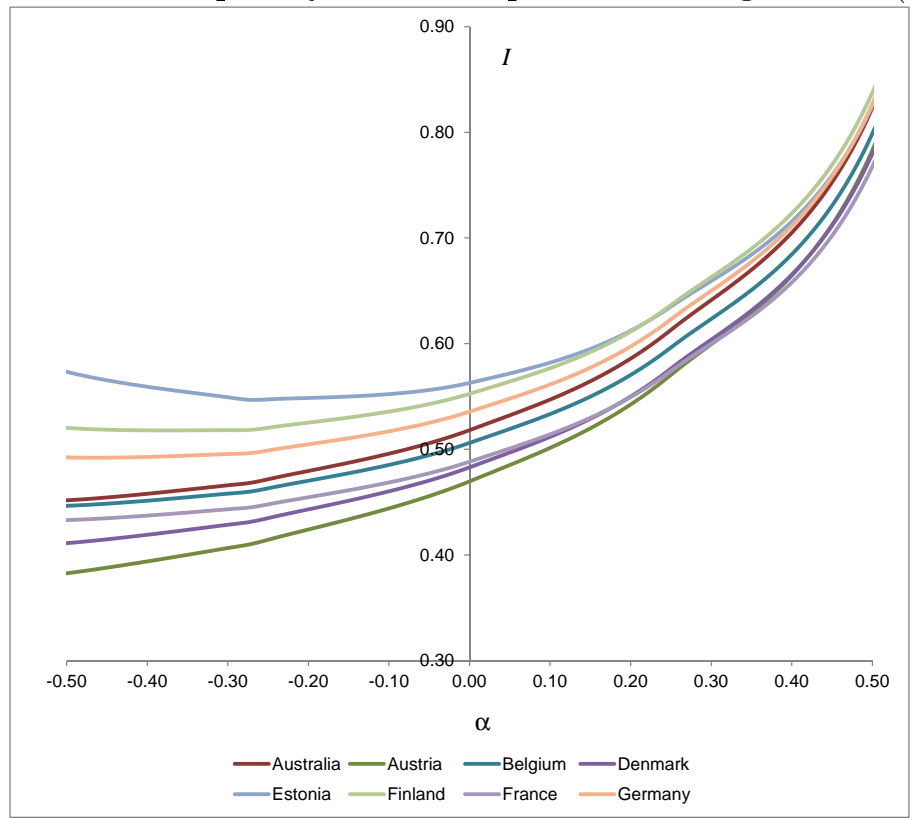


Figure 5: OECD country rankings and $\alpha$. Downward-looking status

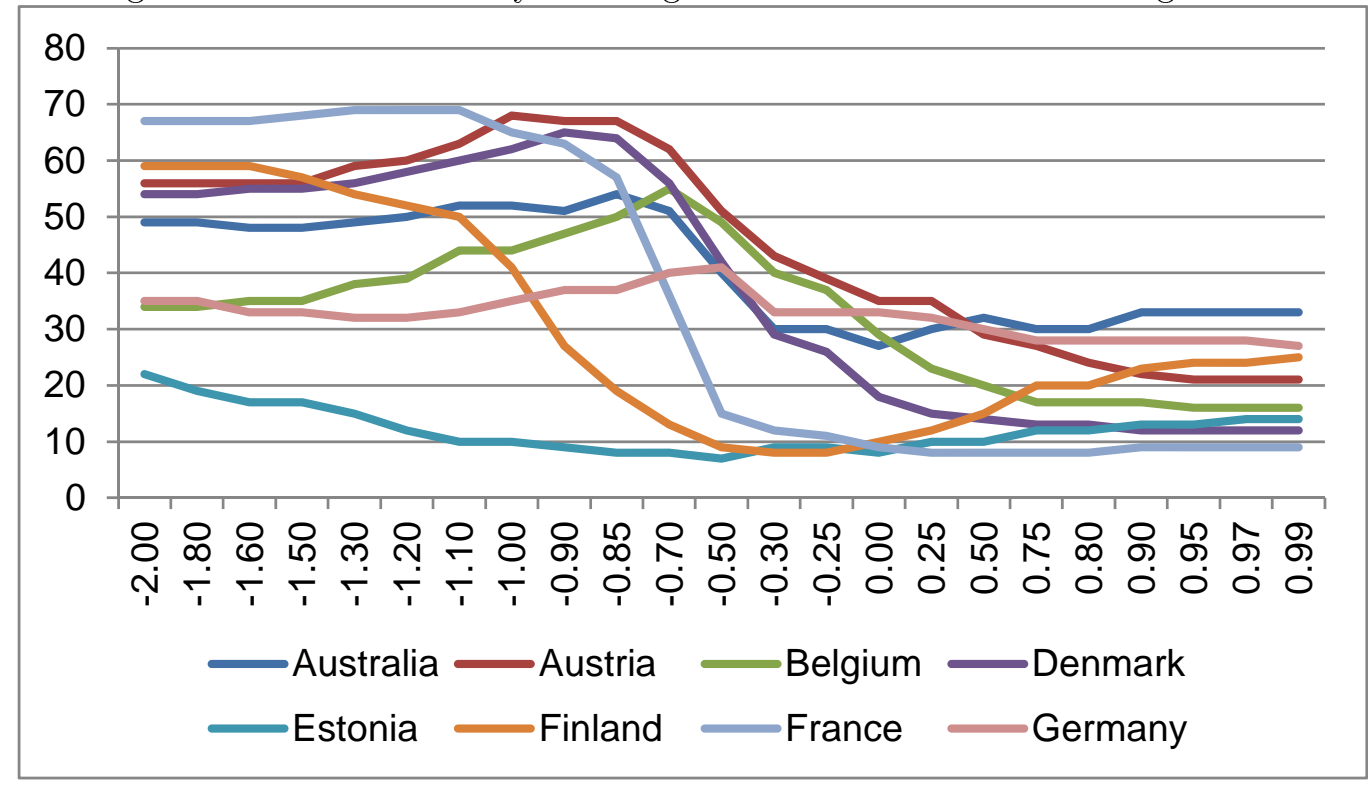

So now let us rank the 70 countries by inequality (such that $1=$ "most equal", $70=$ "most unequal") and plot Figures 5 and 6 , which are snapshots of the orderings generated by the inequality estimates for each value of $\alpha$. In the case of downward-looking status Figure 5 shows a typical pattern for developed countries: the profile is usually downward-sloping ${ }^{4}$ so that as one uses an inequality measure that is progressively less sensitive to observations at the bottom of the status distribution these OECD countries appear lower in the hierarchy. To some extent this is manifest in the case of upwardlooking status (Figure 6): but notice that for the range $\alpha<1 / 2$, the profiles a almost flat. However it is important to note that countries' positions in the health-inequality hierarchy depends crucially on whether a downwardlooking or upward-looking status concept is adopted: contrast the ranking positions of Australia and Estonia within the six-country grouping depicted in Figures 5 and 6.

To throw further light on this in Table 1 we provide the association between upward and downward status. As expected, we find that correlations are dramatically different when values of $\alpha$ vary. Findings are suggestive that the highest correlation between rank orders between upward and downwards

\footnotetext{
${ }^{4}$ Not all OECD profiles follow this pattern - see the discussion of Figure 7 below.
} 
Figure 6: OECD country rankings and $\alpha$. Upward-looking status

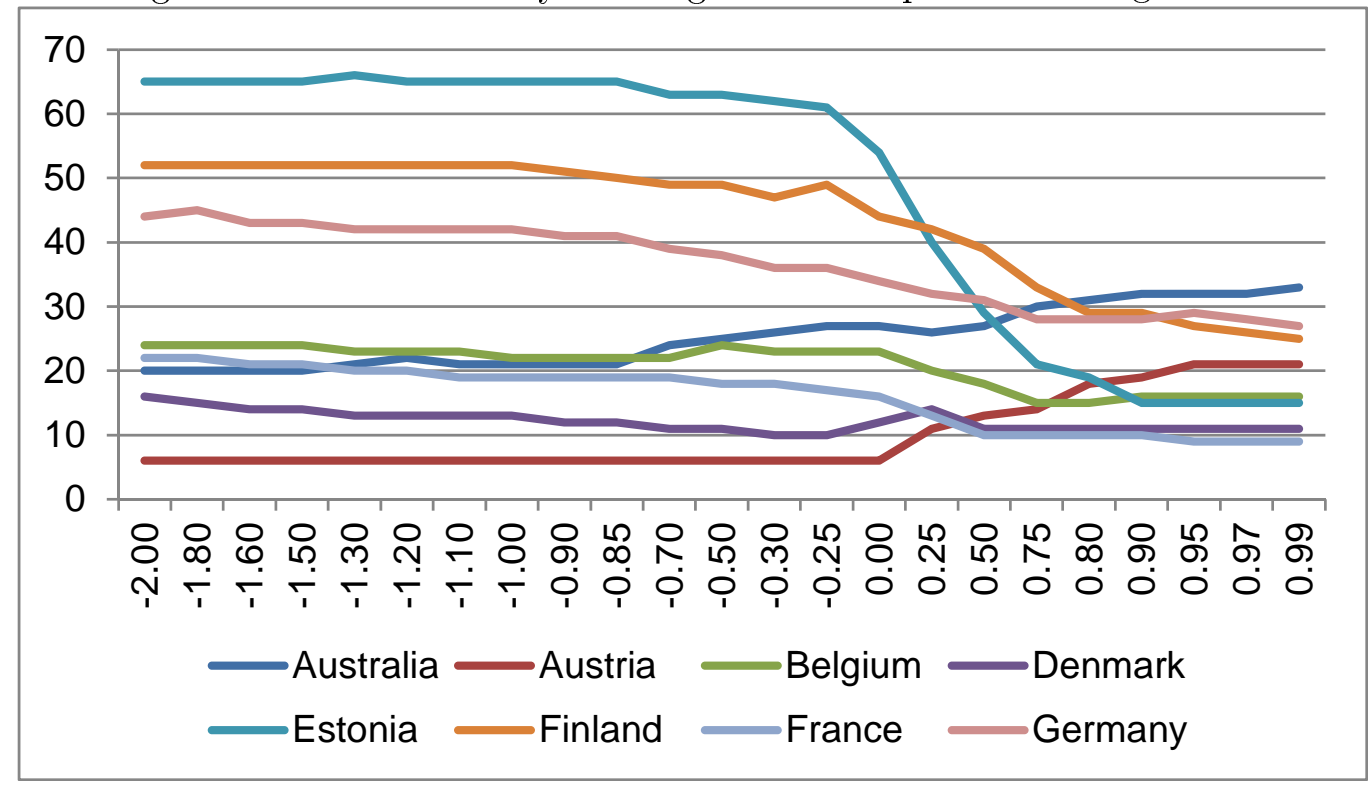

Table 1: Rank-order correlation between upward and downward status inequality for different values of $\alpha$

\begin{tabular}{rc} 
sensitivity & correlation \\
parameter $\alpha$ & $\rho$ \\
\hline-2 & -0.3436 \\
-1 & -0.7997 \\
-0.5 & -0.5265 \\
0 & 0.3354 \\
0.5 & 0.8334 \\
0.99 & 0.9718
\end{tabular}

Note: Significant at $5 \%$ level 
Figure 7: Rerankings of Nordic countries: Downward-looking status

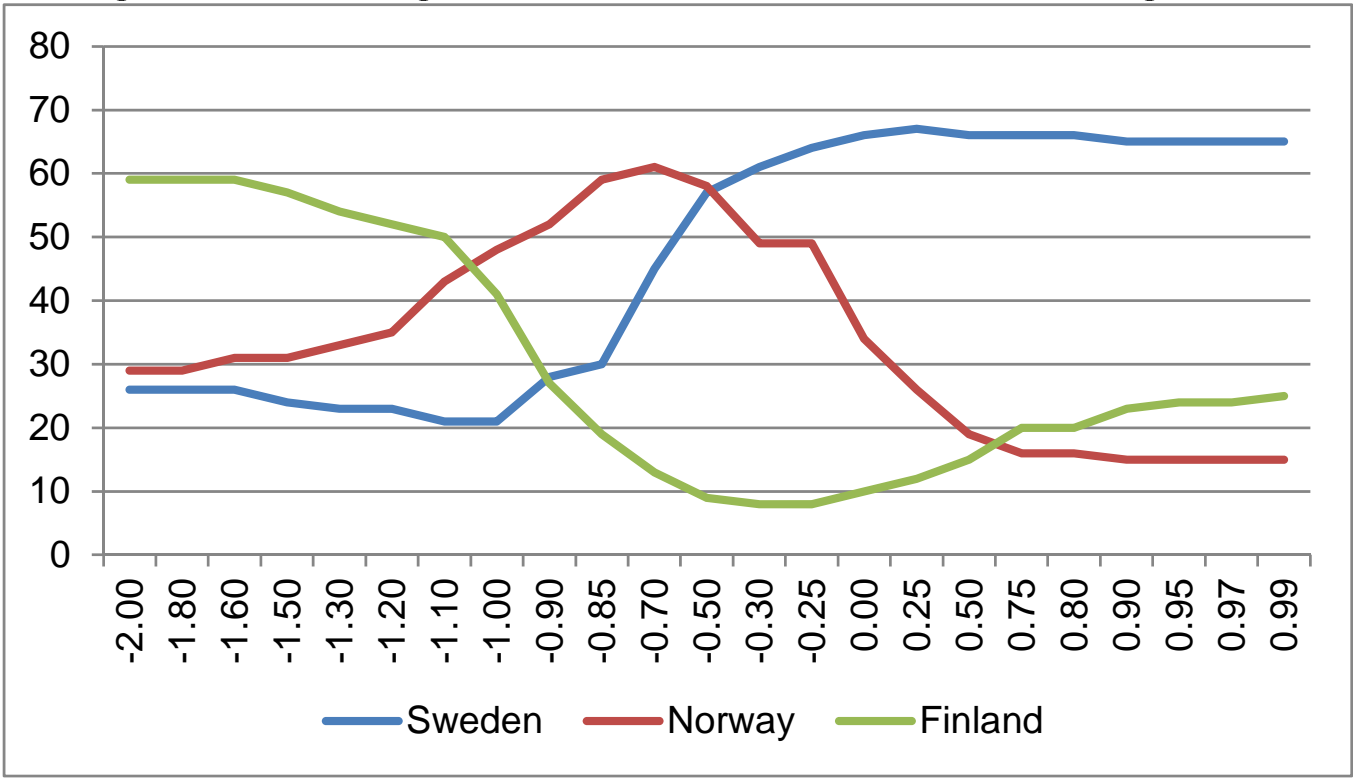

status takes place at a value of $\alpha=0.99$ and the highest inverse correlation for $\alpha=-1$. As expected, we find a positive correlation for positive $\alpha$, and a negative correlation for negative $\alpha$. For an $\alpha=0$ we find a modest but significant correlation of 0.33 , which suggests that the adoption of a downward or upward status perspective does indeed exert a rather significant difference in ranking estimates.

\subsection{The role of inequality sensitivity}

We now turn to a closer examination of the effect of the choice of the inequality-sensitivity parameter, keeping the status concept fixed. In some cases the reranking with $\alpha$ is remarkable; indeed we have seen this in the case of downward-looking status for the six OECD countries depicted in Figure 5 A further nice example is shown in Figure 7: consider the question, for downward-looking status which is the most unequal Nordic country? Figure 7 shows that it depends crucially on the sensitivity index: for large and negative values of $\alpha$ Finland is the most unequal, for positive values $\alpha$ it is Sweden, and for intermediate values it is Norway.

A more formal method of investigating whether the association of country 
Table 2: Rank Order correlation between order from different values of $\alpha$. Downward-looking status

\begin{tabular}{rrrrrrrr} 
& & & & $\alpha$ & & & \\
& & -2 & -1 & -0.5 & 0 & 0.5 & 0.99 \\
\cline { 3 - 9 }$\alpha$ & -2 & 1 & & & & & \\
& -1 & 0.8484 & 1 & & & & \\
& -0.5 & -0.2803 & 0.1388 & 1 & & & \\
& 0 & -0.6475 & -0.4059 & 0.7478 & 1 & & \\
& 0.5 & -0.6566 & -0.5569 & 0.5196 & 0.9365 & 1 & \\
& 0.99 & -0.6420 & -0.6031 & 0.4223 & 0.8860 & 0.9886 & 1
\end{tabular}

Note: Significant at $5 \%$ level

Table 3: Rank Order correlation between order from different values of $\alpha$. Upward-looking status

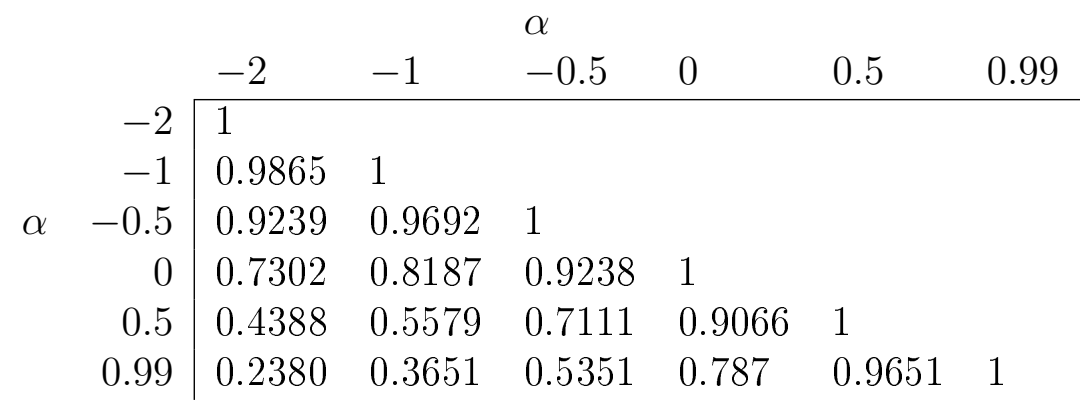

Note: Significant at $5 \%$ level

health inequality rank orders varies with $\alpha$ would be simply to use a measure of rank order correlation. Table 2 presents the Spearman correlation coefficient for downward-looking status. As expected, changes in the values of $\alpha$ induce changes in the ranking of countries based on their health inequalities. In Table 2 we find high correlations when $\alpha$ is positive. As expected turning from a positive $\alpha$ to a negative $\alpha$ flips the sign of the correlation coefficient with the exception of values close a value of $\alpha=0$, where we still find positive correlations.

In Table 3 we follow the same strategy as before for upward looking status, yet in this case we find positive correlation coefficients irrespective of 
Figure 8: Central and East Asian rankings and $\alpha$. Downward-looking status

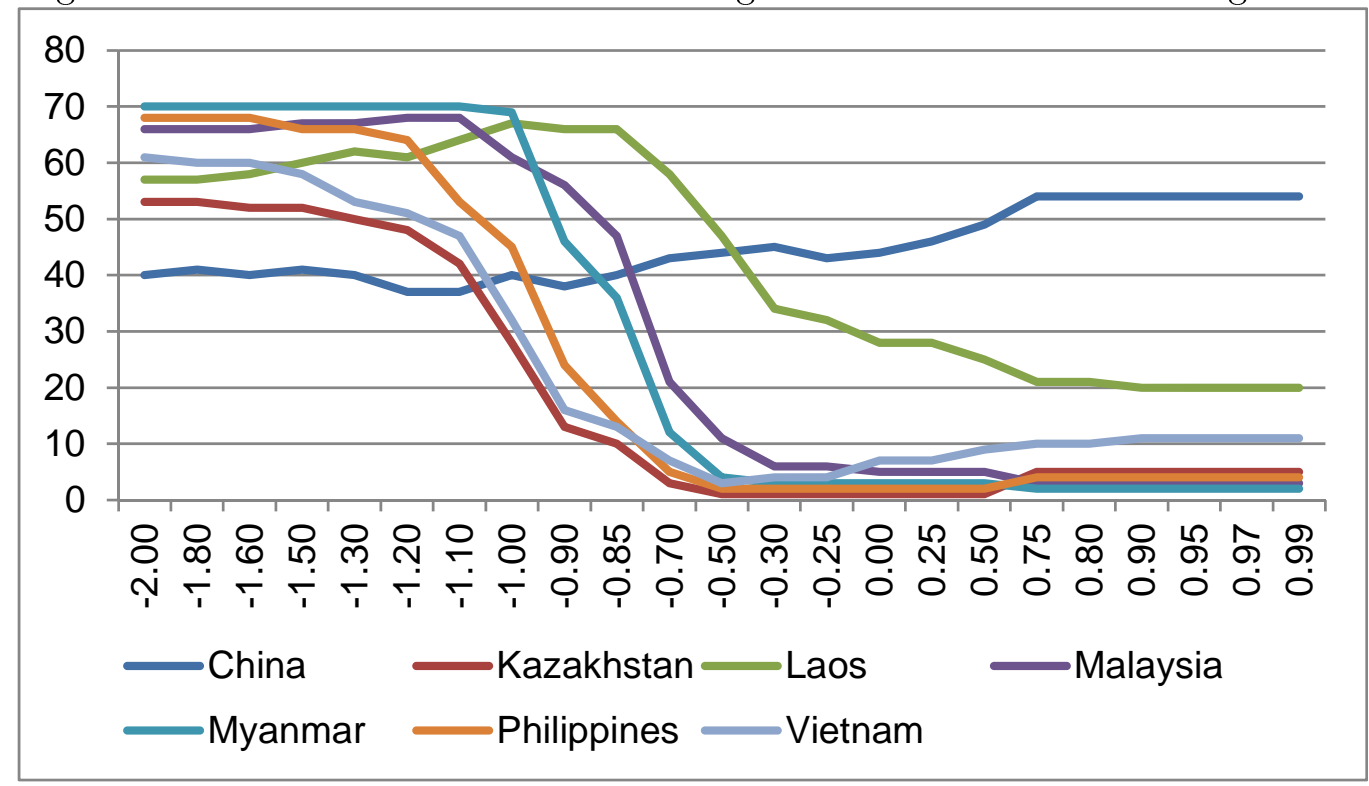

$\alpha$, and generally we find high correlation coefficients except when rankings for extreme values of $\alpha$ are correlated. Based on this data, we can conclude that upward measures of status appear to be less sensitive to changes in $\alpha$ that downward measures of status.

\subsection{Regional patterns}

In addition, we find that inequality-based rankings do exhibit a specific regional mappings, although they do not reflect clear cut or appreciable differences in how health systems are funded and organised. Instead, rankings are sensitive to other unobserved effects that merit additional empirical analysis. For example consider the Asian profiles in Figures 8 and 9: the majority of the profiles are similar to the typical OECD pattern; the major exceptions are the two Asian giants, China and India, where the ranking increases with $\alpha$. These countries profiles are similar to those typical of North and Central Africa (Figure 10) where as $\alpha$ increases towards 1 the position in the health-inequality ranking also increases. 
Figure 9: South Asian rankings and $\alpha$. Downward-looking status



Figure 10: North and Central African rankings and $\alpha$. Downward-looking status

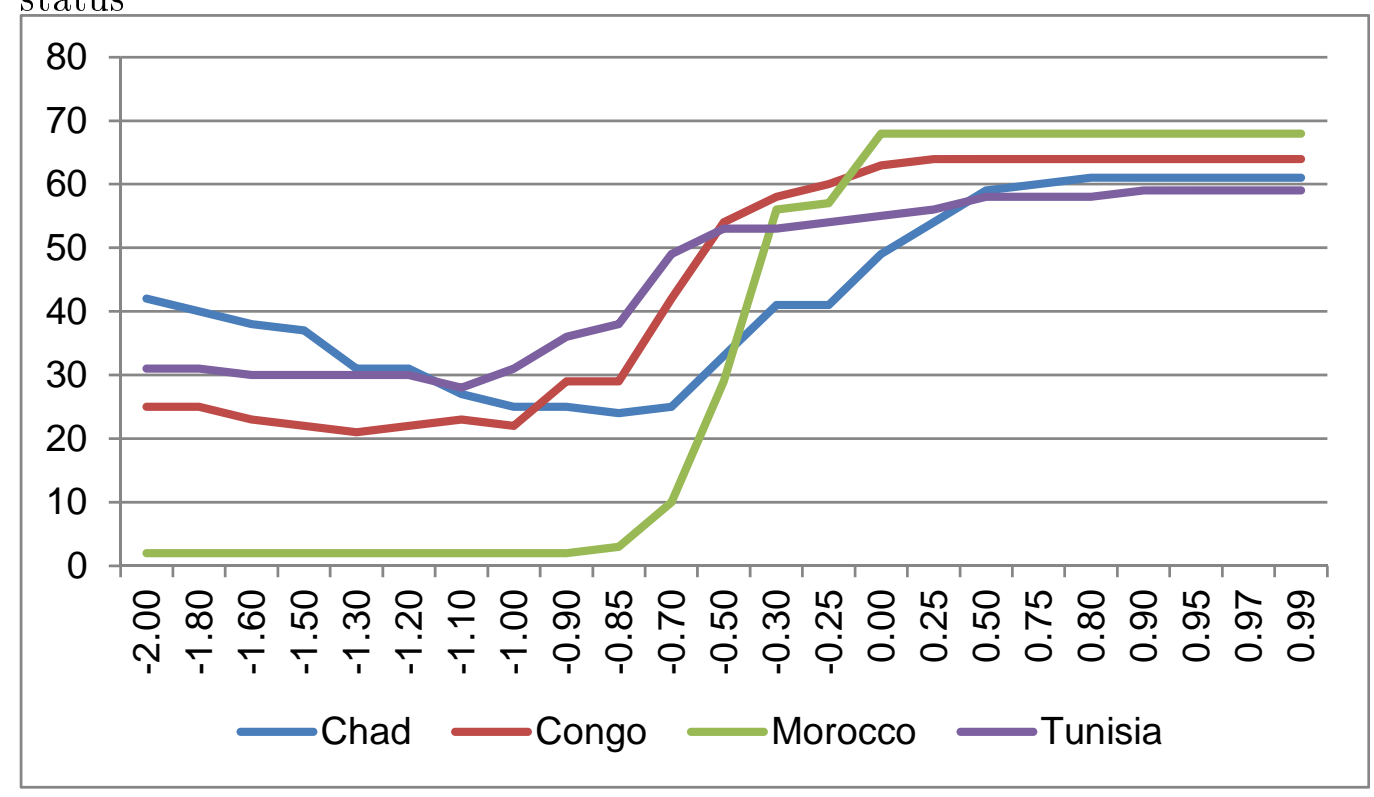




\section{Conclusion}

This paper has shown the applicability of a new method that can be use to undertake international comparisons of health inequality, in line with the aims of the WHO (2000) goals of comparing health systems. We have shown that the methodology can be simply and reliably implemented with categorical data for the purpose of making comparisons of health inequality. From inequality analysis applied in other fields that use cardinal data we know that inequality rankings of different societies can be sensitive to the "inequality aversion" parameter that is used; that applies here too in that changes in the inequality-sensitivity parameter $\alpha$ may have a substantial effect on healthinequality estimates and country rankings. Furthermore, in our case, where categorical data are used, there is an additional consideration: there are two natural methods of quantifying status - downward-looking and upwardlooking - and it is possible that the choice of status concept may also affect country rankings along with choice of $\alpha$.

Our results provide empirical confirmation of the importance of the status concept and the parameter $\alpha$ in ranking health systems. We have shown that the downward or upward status perspective does indeed exert an important difference in ranking estimates. However, whilst changes up the parameter $\alpha$ can invert the country inequality rank-order for downward looking status, we do not find a a flip in the coefficients for upward-looking status. Hence, based on this evidence our tentative recommendation for applied and policy work would be to adopt an upward looking status perspective.

Finally, we have provided some insights on the regional patterns of selfreported health inequality that will form the basis for further research. 


\section{References}

Abul Naga, R. H. and T. Yalcin (2008). Inequality measurement for ordered response health data. Journal of Health Economics 27, 16141625 .

Abul Naga, R. H. and T. Yalcin (2010). Median independent inequality orderings. Technical report, University of Aberdeen Business School.

Allison, R. A. and J. E. Foster (2004). Measuring health inequality using qualitative data. Journal of Health Economics 23, 505-552.

Apouey, B. (2007). Measuring health polarization with self-assessed health data. Health Economics 16(9), 875-894.

Costa-Font, J. and C. Hernández-Quevedo (2012). Measuring inequalities in health: What do we know? what do we need to know? Health Policy 106, 195-206.

Costa-Font, J. and C. Hernández-Quevedo (2013). Inequalities in selfreported health: a meta-regression analysis. LSE Health working paper series in health policy and economics 32/2013, LSE Health and Social Care, London School of Economics and Political Science, London, UK.

Cowell, F. A. and E. Flachaire (2012). Inequality with ordinal data. Public Economics Programme Discussion Paper 16, London School of Economics, http://darp.lse.ac.uk/pdf/IneqOrdinal.pdf.

Cutler, D. and E. Richardson (1997). Measuring the health of the United States population. Brookings Papers on Economic Activity 2, 217-271.

de Barros, R. P., F. Ferreira, J. Chanduvi, and J. Vega (2008). Measuring Inequality of Opportunities in Latin America and the Caribbean. Palgrave Macmillan.

Fleurbaey, M. and E. Schokkaert (2011). Health and health care. In M. Pauly, T. McGuire, and P. Pita-Barros (Eds.), Handbook of Health Economics, Volume 2. North-Holland.

Fonseca, L. and A. M. Jones (2003). Inequalities in self-assessed health in the health survey of England. Equity Working Paper 11, University of York.

Idler, E. and Y. Benyamini (1997). Self-rated health and mortality: a review of 27 community studies. Journal of Health and Social Behaviour 38, 21-37. 
Kapteyn, A., J. P. Smith, and A. Van Soest (2007). Vignettes and selfreports of work disability in the United States and the Netherlands. The American Economic Review 97(1), 461-473.

Kunst, A. E. and J. P. Mackenbach (1994). International variation in the size of mortality differences associated with occupational status. International Journal of Epidemiology 23, 1-9.

LeGrand, J. (1987). Inequalities in health: some international comparisons. European Economic Review 31, 182-191.

Maasoumi, E. (1986). The measurement and decomposition of multidimensional inequality. Econometrica 54, 991-997.

Marmot, M. (2005). Social determinants of health inequalities. Lancet 365(9464), 1099-104.

Oswald, A. J. and S. Wu (2011, November). Well-being across America. The Review of Economics and Statistics 93(4), 1118-1134.

Rice, N., S. Robone, and P. C. Smith (2012). Vignettes and health systems responsiveness in cross-country comparative analyses. Journal of the Royal Statistical Society: Series A (Statistics in Society) 175(2), 337369.

Sen, A. (2002). Health: perception vs. observation. British Medical Journal 324, 860-861.

Stevenson, B. and J. Wolfers (2008). Happiness inequality in the United States. The Journal of Legal Studies 37, S33-S79.

Tsui, K.-Y. (1995). Multidimensional generalizations of the relative and absolute inequality indices: the Atkinson-Kolm-Sen approach. Journal of Economic Theory 67, 251-265.

Van Doorslaer, E. and A. M. Jones (2003). Inequalities in self-reported health: Validation of a new approach to measurement. Journal of Health Economics 22, 61-87.

Wagstaff, A., P. Paci, and E. van Doorslaer (1991). On the measurement of inequalities in health. Social Science and Medicine 33, 545-557.

Wagstaff, A. and E. Van Doorslaer (1994). Measuring inequalities in health in the presence of multiple-category morbidity indicators. Health Economics 3, 281-291. 
Wagstaff, A. and E. van Doorslaer (2000). Equity in health care financing and delivery. In A. J. Culyer and J. P. Newhouse (Eds.), Handbook of Health Economics, pp. 1803-1806. North Holland.

WHO (2000). The World Health Report 2000 - Health systems: Improving performance. Geneva, Switzerland: The World Health Organization.

Yang, Y. (2008). Social inequalities in happiness in the United States, 1972 to 2004: An age-period-cohort analysis. American Sociological Review 73, 204-226.

Zheng, B. (2011). A new approach to measure socioeconomic inequality in health. Journal Of Economic Inequality 9, 555-577.

\section{Appendix}

The data are taken from the the World Health Organization's World Health Survey, which is described here:

http://www.who.int/healthinfo/survey/instruments/en/index.html

In particular, for our categorical variable, we use the responses to one specific question in this survey's collection of individual questions about overall health.

"Q2000: In general, how would you rate your health today? The respondent should answer according to how he/she considers his/her health to be and give his/her best estimate. Both physical and mental health must be taken into consideration."

Table 4 reports the answers to this question across 70 countries. The titles of Columns (1) to (5) give the categories used in the survey: we follow the natural order taking (1) as the best category and (5) as the worst. The total number of respondents is in column (6): if in any row this total exceeds the sum of columns (1) to (5), the difference is attributable to non-response. 
Table 4: Responses in WHS to self-rated health question

\begin{tabular}{rrrrrrrr}
\hline & & $(1)$ & $(2)$ & $(3)$ & $(4)$ & $(5)$ & $(6)$ \\
& Country & Very good & Good & Moderate & Bad & Very Bad & Total \\
1 & Australia & 487 & 775 & 446 & 74 & 11 & 1793 \\
2 & Austria & 423 & 390 & 200 & 36 & 4 & 1053 \\
3 & Bangladesh & 494 & 1949 & 2132 & 741 & 228 & 5544 \\
4 & Belgium & 252 & 487 & 197 & 48 & 11 & 995 \\
5 & Bosnia & 271 & 328 & 279 & 127 & 23 & 1028 \\
6 & Brazil & 715 & 1934 & 1881 & 348 & 119 & 4997 \\
7 & Burkina Faso & 1254 & 2104 & 1137 & 288 & 36 & 4819 \\
8 & Chad & 889 & 1767 & 1371 & 549 & 37 & 4613 \\
9 & China & 982 & 1485 & 1215 & 277 & 34 & 3993 \\
10 & Comoros & 312 & 631 & 523 & 261 & 30 & 1757 \\
11 & Congo & 693 & 550 & 693 & 252 & 33 & 2221 \\
12 & Côte d'Ivoire & 661 & 1215 & 955 & 266 & 21 & 3118 \\
13 & Croatia & 200 & 302 & 312 & 132 & 43 & 989 \\
14 & Czech & 160 & 350 & 311 & 90 & 19 & 930 \\
15 & Denmark & 320 & 472 & 166 & 40 & 4 & 1002 \\
16 & Dominican & 722 & 1806 & 1560 & 397 & 34 & 4519 \\
17 & Ecuador & 650 & 1945 & 1569 & 378 & 53 & 4595 \\
18 & Estonia & 70 & 293 & 499 & 134 & 16 & 1012 \\
19 & Ethiopia & 2138 & 1549 & 972 & 220 & 47 & 4926 \\
20 & Finland & 158 & 395 & 391 & 64 & 3 & 1011 \\
21 & France & 255 & 525 & 192 & 34 & 2 & 1008 \\
22 & Georgia & 265 & 778 & 1111 & 476 & 125 & 2755 \\
23 & Germany & 229 & 582 & 343 & 85 & 13 & 1252 \\
24 & Ghana & 1379 & 1433 & 830 & 234 & 46 & 3922 \\
25 & Greece & 347 & 325 & 246 & 63 & 19 & 1000 \\
26 & Guatemala & 730 & 1790 & 1747 & 472 & 24 & 4763 \\
27 & Hungary & 139 & 579 & 503 & 155 & 34 & 1410 \\
28 & India & 2159 & 3577 & 2616 & 1311 & 202 & 9865 \\
29 & Ireland & 366 & 257 & 101 & 29 & 5 & 758 \\
30 & Israel & 519 & 405 & 234 & 40 & 23 & 1221 \\
31 & Italy & 182 & 449 & 305 & 46 & 15 & 997 \\
32 & Kazakhstan & 265 & 1894 & 2088 & 231 & 18 & 4496 \\
33 & Kenya & 1115 & 1798 & 1144 & 309 & 40 & 4406 \\
34 & Lao & 1787 & 2005 & 906 & 168 & 17 & 4883
\end{tabular}




\begin{tabular}{|c|c|c|c|c|c|c|c|}
\hline \multicolumn{8}{|c|}{...continued } \\
\hline & Country & Very good & Good & Moderate & Bad & Very Bad & Total \\
\hline 35 & Latvia & 35 & 244 & 390 & 155 & 32 & 856 \\
\hline 36 & Luxembourg & 164 & 346 & 155 & 33 & 2 & 700 \\
\hline 37 & Malawi & 2855 & 1334 & 838 & 231 & 33 & 5291 \\
\hline 38 & Malaysia & 1194 & 3495 & 1111 & 204 & 12 & 6016 \\
\hline 39 & Mali & 1334 & 1526 & 895 & 266 & 11 & 4032 \\
\hline 40 & Mauritania & 941 & 1672 & 1024 & 154 & 9 & 3800 \\
\hline 41 & Mauritius & 850 & 1677 & 827 & 427 & 104 & 3885 \\
\hline 42 & Mexico & 7193 & 18112 & 11221 & 2002 & 218 & 38746 \\
\hline 43 & Morocco & 598 & 1454 & 1754 & 821 & 372 & 4999 \\
\hline 44 & Myanmar & 1215 & 3412 & 1100 & 157 & 2 & 5886 \\
\hline 45 & Namibia & 1622 & 1249 & 863 & 204 & 45 & 3983 \\
\hline 46 & Nepal & 1455 & 3908 & 2505 & 767 & 53 & 8688 \\
\hline 47 & Netherlands & 189 & 640 & 214 & 41 & 2 & 1086 \\
\hline 48 & Norway & 314 & 456 & 140 & 46 & 13 & 969 \\
\hline 49 & Pakistan & 1770 & 2996 & 1315 & 263 & 25 & 6369 \\
\hline 50 & Paraguay & 1700 & 1920 & 1370 & 133 & 16 & 5139 \\
\hline 51 & Philippines & 817 & 5127 & 3759 & 354 & 19 & 10076 \\
\hline 52 & Portugal & 62 & 342 & 390 & 180 & 55 & 1029 \\
\hline 53 & Russia & 261 & 1102 & 2192 & 770 & 91 & 4416 \\
\hline 54 & Senegal & 646 & 1028 & 984 & 217 & 24 & 2899 \\
\hline 55 & Slovakia & 400 & 798 & 506 & 94 & 17 & 1815 \\
\hline 56 & Slovenia & 90 & 238 & 193 & 53 & 9 & 583 \\
\hline 57 & South Africa & 837 & 865 & 467 & 129 & 42 & 2340 \\
\hline 58 & Spain & 1051 & 2984 & 1689 & 502 & 117 & 6343 \\
\hline 59 & Sri Lanka & 1844 & 3019 & 1535 & 298 & 22 & 6718 \\
\hline 60 & Swaziland & 198 & 451 & 508 & 676 & 236 & 2069 \\
\hline 61 & Sweden & 262 & 354 & 235 & 133 & 14 & 998 \\
\hline 62 & Tunisia & 1236 & 1850 & 1476 & 411 & 58 & 5031 \\
\hline 63 & Turkey & 1301 & 4809 & 4035 & 869 & 189 & 11203 \\
\hline 64 & $\mathrm{UAE}$ & 536 & 472 & 146 & 18 & 6 & 1178 \\
\hline 65 & UK & 318 & 498 & 278 & 82 & 17 & 1193 \\
\hline 66 & Ukraine & 129 & 659 & 1364 & 594 & 103 & 2849 \\
\hline 67 & Uruguay & 725 & 1632 & 547 & 63 & 9 & 2976 \\
\hline 68 & Vietnam & 398 & 1368 & 1489 & 225 & 10 & 3490 \\
\hline 69 & Zambia & 1436 & 1292 & 816 & 228 & 39 & 3811 \\
\hline 70 & Zimbabwe & 837 & 1263 & 1501 & 385 & 65 & 4051 \\
\hline
\end{tabular}

